\title{
Primary Sjogren Syndrome
}

National Cancer Institute

\section{Source}

National Cancer Institute. Primary Sjogren Syndrome. NCI Thesaurus. Code C116985.

Sjogren syndrome without a concomitant systemic autoimmune disorder. 